\title{
Análise da distribuição e cobertura do NASF na IV Macrorregião de Saúde do Estado de Pernambuco.
}

\author{
Alaine Santos Parente ${ }^{1}$; Fabiola Olinda de Souza Mesquita ${ }^{2}$; Sued Sheila Sarmento ${ }^{3}$
}

Resumo: O estudo apresentou como objetivo analisar a distribuição e cobertura do Núcleo de apoio à Saúde da Família (NASF) na IV Macrorregião de saúde do estado de Pernambuco. Foi realizado estudo descritivo de abordagem quantitativa a partir de dados secundários do Cadastro Nacional de Estabelecimentos de Saúde (CNES). Observou-se que as regionais VII, VIII e IX possuem 293 ESF e 32 equipes de NASF nas modalidades 1 e 2. Foi constatada ausência de implantação desse serviço em alguns municípios, bem como excesso de ESF vinculadas em outros. A partir dos dados, observou-se que o número de NASF implantados é insuficiente para apoiar as ESF existentes. Dessa forma, verifica-se que há a necessidade de expansão dessas equipes com vistas a adequação das normas preconizadas pelo Ministério da Saúde.

Palavras-chave: Atenção primária à saúde. Saúde da família. Cobertura de serviços públicos de saúde.

\section{Analysis of distribution and NASF coverage in the macro-IV Pernambuco state health.}

\begin{abstract}
The study presented as objective to analyze the distribution and coverage of the Family Health to support core (NASF) in IV Macroregion health of Pernambuco state. Descriptive study was conducted a quantitative approach based on secondary data from the National Health Facilities (CNES). It was noted that regional VII, VIII and IX have 293 ESF and 32 teams NASF in modes 1 and 2. It was found lack of implementation of the service in some municipalities, as well as excess ESF linked in others. From the data, it was observed that the number of implanted NASF is insufficient to support existing ESF. Thus, it appears that there is a need for expansion of these teams with a view to adjusting the provisions recommended by the Ministry of Health.
\end{abstract}

Key-words: Primary health care. Family health. State health care coverage.

\section{Introdução}

O Sistema Único de Saúde (SUS) tem enfrentado inúmeros desafios para sua consolidação. Entre os desafios destaca-se a dificuldade em estabelecer uma rede regionalizada de serviços que atenda as necessidades da população (TOMASI; RIZZOTTO, 2013).

\footnotetext{
${ }^{1}$ Graduada em Fisioterapia pela Faculdade Santa Maria- Cajazeiras- PB; Especialização em Gestão em Saúde pela Universidade Federal do Vale do São Francisco (UNIVASF). E-mail: alainefisio@ hotmail.com.

${ }^{2}$ Graduada em Odontologia pela Universidade Federal de Pernambuco; Especialização em Gestão em Saúde pela Universidade Federal do Vale do São Francisco (UNIVASF). E-mail: fabiolaolinda@ yahoo.com.br.

${ }^{3}$ Graduada em Enfermagem pela Universidade Estadual da Paraíba; Mestrado em Psicologia pela Universidade Federal do Espírito Santo; Email: sued.sheila@gmail.com.
} 
A organização desse sistema requer a construção de redes integradas em saúde que comportem a atenção as doenças agudas e crônicas apresentadas no Brasil. O tema sobre as Redes de atenção a Saúde (RAS) não é novo no país e já estava inserido desde a Constituição Federal de 1988, no Artigo 198, o qual menciona que as ações e serviços públicos de saúde integram uma rede regionalizada e hierarquizada (MENDES, 2014).

A configuração de redes pressupõe a construção de relações niveladas entre os diferentes serviços que passam a ser interdependentes e possuir canais de comunicação entre si. Possuindo uma região como base territorial e a Atenção Primária à Saúde (APS) como o centro de comunicação, essas redes podem ser estabelecidas por temas de atenção que apresentam similaridades no modo de organizar o cuidado aos usuários (MAGALHÃES JÚNIOR, 2014).

A sua construção sinaliza a preocupação em superar a concepção de hierarquização de serviços por níveis de complexidade para uma organização poliárquica em que todos os serviços são igualmente importantes e interdependentes entre si (MENDES, 2011). Essas redes deverão ser compostas por: um centro de comunicação, a APS, pontos de atenção secundários e terciários, sistemas logísticos e de apoio. A APS corresponde a um elemento essencial configurando-se como um centro comunicador e ordenador do cuidado estabelecendo fluxos de referência e contrarreferência no SUS (MENDES, 2010).

Com o intuito de apoiar a consolidação e o fortalecimento da atenção básica no Brasil, o Ministério da Saúde (MS) instituiu no ano de 2008 o Núcleo de Apoio à Saúde da Família (NASF) (BRASIL, 2008). Corresponde a um dispositivo estratégico na RAS para a melhoria da qualidade da atenção básica através da atuação conjunta e articulada com a Estratégia de Saúde da Família (ESF) ampliando a capacidade de resposta a maioria dos problemas de saúde da população e a resolubilidade da atenção primária (BRASIL, 2014).

O NASF é formado por equipes multiprofissionais que deverão ser constituídas a partir das necessidades de cada território. Pode ser considerado como "retaguarda" da ESF, por atuar em conjunto com essas equipes, compartilhando com elas saberes e práticas de saúde (NASCIMENTO; OLIVEIRA, 2010). Em sua composição estão previstos os seguintes 
profissionais: professores de educação física, fisioterapeutas, fonoaudiólogos, nutricionistas, psicólogos, terapeutas ocupacionais, médicos, etc (GONÇALVES et al, 2015).

O desenvolvimento da proposta de trabalho do NASF baseia-se na atuação entre uma equipe de referência e uma equipe de apoio. Esse tipo de arranjo busca construir uma responsabilização compartilhada entre as equipes objetivando ampliar as possibilidades de construção de vínculos entre profissionais e usuários. Dessa forma, propõe-se estabelecer um modelo de atenção que supere a lógica fragmentada em saúde em direção a corresponsabilização do cuidado (ANJOS et al, 2013). É essencial que para a agenda cotidiana do trabalho, os profissionais tenham clareza sobre o papel que desempenham, contribuindo para fortalecer a prática de apoio matricial e construção compartilhada (FITIIPALDI; ROMANO; BARROS, 2015).

Esse serviço vem se estruturando gradativamente a partir da publicação de vários documentos oficiais do Ministério da Saúde (MS). Inicialmente foram publicados dois documentos importantes: a Portaria GM/MS n $154 / 2008$, que cria o NASF, e o Caderno de Atenção Básica $\mathrm{n}^{\circ}$ 27, que apresenta as diretrizes, a organização e o desenvolvimento do processo de trabalho do mesmo (BRASIL, 2008, BRASIL, 2009).

Após 3 anos de sua criação, outras portarias surgem no intuito de revisar os parâmetros de vinculação e definir as fontes de financiamento, entre elas: a Portaria 2.488/2011 a qual aprova a Política Nacional de Atenção Básica (PNAB) e a portaria 3.124/2012 que redefine os parâmetros de vinculação das equipes (BRASIL, 2011; BRASIL, 2012). Essas redefinições sinalizam a preocupação do Ministério em consolidar a proposta do NASF na rede.

Apesar de ser criado em 2008, ainda está em processo de construção e, como o antigo Programa Saúde da Família (PSF), atual Estratégia de Saúde da Família (ESF), está caminhando aos poucos, até que a população e a ESF entendam a sua função (LIMA, 2013).

O primeiro documento oficial que dispõe sobre essas equipes, propôs a conformação de duas modalidades de NASF: 1 (um) e 2 (dois). A primeira modalidade poderia estar vinculada a no mínimo 8 e no máximo 20 ESF, com exceção dos municípios com menos de 
100.000 habitantes dos Estados da Região Norte, que poderiam realizar suas atividades vinculado a, no mínimo, 5 e no máximo, a 20 ESF. Já a modalidade 2 deveria estar vinculado a no mínimo 3 ESF (BRASIL, 2008). Essa proposta de configuração não contemplava a maioria dos municípios brasileiros, especialmente aqueles de pequeno porte que possuíam poucas ESF.

Em 2012, visando a ampliação dessas equipes e um melhor desenvolvimento do seu processo de trabalho, o MS publicou portaria que estabelece a revisão dos parâmetros de vinculação, criando ainda uma terceira modalidade: O NASF 3. A partir desta, ficou instituído que cada NASF 1 deverá estar vinculado a no mínimo 5 (cinco) e a no máximo 9 (nove) ESF e/ou equipes de Atenção Básica para populações específicas (consultórios na rua, equipes ribeirinhas e fluviais); o NASF 2 a no mínimo 3 (três) e a no máximo, 4 (quatro) equipes e o NASF 3 de uma a duas ESF (BRASIL, 2012).

Desde a sua criação os municípios vêm progressivamente aderindo a essa proposta com o objetivo de oferecer retaguarda especializada para as equipes de atenção básica. Apesar do seu crescimento, poucas pesquisas analisam e demonstram a sua distribuição e cobertura nos diferentes municípios do país. Grande parte dos estudos centram-se na análise do processo de trabalho e operacionalização das ações, destacando os seus desafios e potencialidades. Dessa forma, aponta-se a necessidade da realização de estudos que demonstrem o grau de implantação dessa estratégia.

Nesse sentido, Santos; Benedetti (2012) complementam a importância em conhecer e acompanhar o desenvolvimento desse serviço possibilitando a reavaliação do grau de investimento para a concretização desta proposta, bem como as mudanças necessárias na qualificação da gestão e dos recursos humanos para o SUS.

Portanto, essa pesquisa apresenta como objetivo analisar a distribuição e cobertura do NASF na IV Macrorregião de Saúde do Estado de Pernambuco. Como objetivos específicos o estudo pretendeu: descrever o número de equipes de NASF e equipes de saúde da família implantadas; verificar a cobertura, apresentar o teto de implantação e potencial de expansão do NASF na IV Macrorregião de saúde do estado de Pernambuco. 


\section{Metodologia}

Trata-se de um estudo descritivo com abordagem quantitativa realizado a partir de dados secundários do Departamento de Informática do SUS (DATASUS)/ Ministério da Saúde (MS), em específico do Cadastro Nacional dos Estabelecimentos de Saúde (CNES).

A utilização dos dados secundários foi necessária para obtenção do número de equipes de NASF e equipes de saúde da família cadastradas por município da IV Macrorregião de saúde do estado de Pernambuco.

De acordo com o plano diretor de regionalização (2011), Pernambuco possui 04 macrorregiões e 12 regiões de Saúde, com sede nas seguintes cidades: I Geres (Recife); II (Limoeiro); III (Palmares); IV (Caruaru); V (Garanhuns); VI (Arcoverde); VII (Salgueiro); VIII (Petrolina); IX (Ouricuri); X ( Afogados da ingazeira); XI (Serra talhada) e XII (Goiana). A IV Macrorregião corresponde ao Vale do São Francisco e Araripe e é composta pelas regionais VII, VIII e IX (PERNAMBUCO, 2011).

As variáveis selecionadas para a pesquisa foram: número de equipes de NASF e Equipes de Saúde da família. Através da obtenção desses dados foi possível realizar o cálculo do teto de implantação, cobertura e potencial de expansão do NASF.

Para definição do teto de implantação foram utilizadas as instruções do caderno de atenção básica $\mathrm{n}^{0}$ 39. De acordo com esse documento para a definição do território a ser coberto pelo NASF deve-se considerar o número de equipes de $\mathrm{AB}$ no município, sua disposição na Rede de atenção a saúde (RAS) e o número de NASF a serem implantados (bem como sua modalidade). De forma prática, divide-se o número total de ESF ativas no CNES pelo número de equipes que pode ser vinculado a cada modalidade de NASF. A partir desse cálculo, obtém-se o quantitativo de equipes que poderão ser implantados no município com financiamento federal, considerando os parâmetros para cada modalidade conforme a Portaria $\mathrm{n}^{\mathrm{o}} 3.124$, de 28 de dezembro de 2012 (BRASIL, 2014).

A avaliação da cobertura foi realizada mediante o cálculo do número de NASF cadastrados no CNES sobre o teto de implantação dessas equipes multiplicado por 100 . Por 
fim, o potencial de expansão corresponde a diferença entre o teto de implantação e número de equipes de NASF cadastradas.

O cálculo foi realizado considerando apenas a modalidade 1, pois de acordo com o MS a implantação de mais de uma modalidade de NASF concomitantemente no mesmo município não receberá incentivo financeiro federal, sendo que o número de NASF 2 e NASF 3 que fará jus ao recebimento desse incentivo é de apenas 1 (BRASIL, 2012).

Os dados foram coletados considerando a competência de fevereiro de 2016 pois correspondeu a competência disponível no CNES no período de coleta de dados. Após a coleta foi realizada análise descritiva através da apresentação do número absoluto de equipes, bem como do percentual de cobertura utilizando o programa Microsoft Excel 2007.

Com o intuito de facilitar a visualização das informações foram construídas tabelas para exposição das 3 regionais escolhidas, as quais foram descritas as variáveis de análise por município de cada região de saúde.

Para análise dos dados foi levado em consideração as instruções do MS, o qual orienta que a definição do número de equipes que serão vinculadas a cada NASF, envolve uma organização que contemple o menor número possível de equipes de atenção básica vinculadas, facilitando o processo de trabalho compartilhado entre esses serviços (BRASIL, 2014). A análise final consistiu em correlacionar os dados obtidos com o referencial teórico a cerca da temática.

Por tratar-se de estudo com utilização de dados secundários não foi necessária a submissão do projeto ao Comitê de Ética em Pesquisa, uma vez que se tratam de informações de acesso público disponibilizadas na internet. 


\section{Resultados}

A partir do estudo observou-se o número de equipes NASF e ESF, o teto de implantação, cobertura e potencial de expansão das equipes de NASF por município da IV Macrorregião de saúde do Estado de Pernambuco.

Considerando os dados da tabela 1 visualiza-se que a IV Macrorregião de Saúde, composta pelas regionais VII, VIII e IX, possuem 293 ESF e 32 equipes de NASF na modalidade 1 e 2 . Observa-se que não existem NASF na modalidade 3 implantados.

O NASF 3 é uma modalidade adequada a realidade de pequenos municípios que possuem até 2 equipes de saúde da família. Também é possível observar a distribuição de equipes por município das referidas regiões de saúde. Observa-se a ausência de implantação do NASF nos municípios de Belém de São Francisco, Terra Nova, Orocó, e Moreilândia.

Em relação aos parâmetros de vinculação do NASF as ESF, observa-se que em alguns municípios o número de ESF para cada NASF é superior a recomendação do Ministério da Saúde, a exemplo de: Cedro, Mirandiba, Salgueiro, Afrânio, Cabrobó, Dormentes, Lagoa Grande, Petrolina, Bodocó, Ipubi e Trindade.

Nos municípios de Cedro, Mirandiba, Afrânio e Dormentes deveria ocorrer a adequação do NASF na modalidade 2 para 1. Em Salgueiro poderiam ser implantados 2 NASF considerando o parâmetro mínimo de recomendação do MS (1 NASF para 5 ESF) ou apenas mais 1 NASF considerando o parâmetro máximo (1 NASF para 9 ESF). Também existe a necessidade de implantação de novos NASF nos municípios de Cabrobó, Lagoa Grande, Petrolina, Bodocó, Ipubi e Trindade. Essa distribuição permitiria o suporte a todas as ESF existentes no território (tabela 1). 
Tabela 1- Número de equipes de NASF e ESF por município na VII, VIII e IX Região de Saúde do estado de Pernambuco na competência fevereiro de 2016.

\begin{tabular}{|c|c|c|c|c|}
\hline $\begin{array}{c}\text { REGIONAL } \\
\text { VII (Salgueiro) }\end{array}$ & $\begin{array}{l}\mathbf{N}^{\mathbf{d}} \text { de } \\
\text { ESF }\end{array}$ & NASF 1 & NASF 2 & NASF 3 \\
\hline Belém de São Francisco & 7 & 0 & 0 & 0 \\
\hline Cedro & 5 & 0 & 1 & 0 \\
\hline Mirandiba & 5 & 0 & 1 & 0 \\
\hline Salgueiro & 18 & 1 & 0 & 0 \\
\hline Serrita & 8 & 1 & 0 & 0 \\
\hline Terra Nova & 4 & 0 & 0 & 0 \\
\hline Verdejante & 4 & 0 & 1 & 0 \\
\hline Total & 51 & 2 & 3 & $\mathbf{0}$ \\
\hline \multicolumn{5}{|l|}{ VIII (Petrolina) } \\
\hline Afrânio & 6 & 0 & 1 & 0 \\
\hline Cabrobó & 11 & 1 & 0 & 0 \\
\hline Dormentes & 5 & 0 & 1 & 0 \\
\hline Lagoa Grande & 10 & 1 & 0 & 0 \\
\hline Orocó & 4 & 0 & 0 & 0 \\
\hline Petrolina & 83 & 8 & 0 & 0 \\
\hline Santa Maria da Boa Vista & 9 & 1 & 0 & 0 \\
\hline Total & 128 & 11 & 2 & $\mathbf{0}$ \\
\hline \multicolumn{5}{|l|}{ IX (Ouricuri) } \\
\hline Araripina & 17 & 2 & 0 & 0 \\
\hline Bodocó & 11 & 1 & 0 & 0 \\
\hline Exu & 13 & 2 & 0 & 0 \\
\hline Granito & 3 & 0 & 1 & 0 \\
\hline Ipubi & 11 & 1 & 0 & 0 \\
\hline Moreilândia & 5 & 0 & 0 & 0 \\
\hline Ouricuri & 24 & 3 & 0 & 0 \\
\hline Santa Cruz & 6 & 1 & 0 & 0 \\
\hline Santa Filomena & 5 & 1 & 0 & 0 \\
\hline Trindade & 11 & 1 & 0 & 0 \\
\hline Parnamirim & 8 & 1 & 0 & 0 \\
\hline Total & 114 & 13 & 1 & 0 \\
\hline
\end{tabular}

Fonte: CNES/DATASUS, 2016.

Nas tabelas 2, 3 e 4 visualiza-se o teto de implantação, cobertura e potencial de expansão de NASF. O teto de implantação corresponde ao número máximo de equipes de NASF que podem ser implantadas com financiamento federal (BRASIL, 2014). Esse cálculo 
é realizado seguindo os parâmetros de vinculação estabelecidos na portaria 3.124 de 28 de dezembro de 2012. O NASF em sua modalidade 1 pode oferecer suporte a no mínimo 5 e no máximo 9 equipes de atenção básica (BRASIL, 2012). Os cálculos foram realizados considerando o parâmetro mínimo representado pelo teto $\mathrm{A}$, bem como parâmetro máximo descrito como teto $\mathrm{B}$.

Observa-se que a VII regional possui uma cobertura de $19,60 \%$ considerando o parâmetro mínimo e de 35,33\% de acordo com o parâmetro máximo recomendado. Dessa forma, percebe-se que o número de NASF implantados parece ser insuficiente para dar suporte a todas as equipes de saúde da família existentes. Em relação ao potencial de expansão verificou-se que podem ser implantadas aproximadamente 10 NASF considerando o teto a ou 5 NASF considerando o teto b (tabela 2).

Tabela 2 - Teto de implantação, cobertura e potencial de expansão do NASF na VII regional de Saúde da IV Macro do estado de Pernambuco na competência fevereiro de 2016.

\begin{tabular}{|c|c|c|c|c|c|c|c|c|}
\hline \multirow[t]{2}{*}{ Município } & \multirow[t]{2}{*}{$\begin{array}{l}N^{0} \text { de } \\
\text { ESF }\end{array}$} & \multirow{2}{*}{$\begin{array}{c}\mathrm{N}^{\circ} \text { de } \\
\text { NASF } \\
1\end{array}$} & \multirow{2}{*}{$\begin{array}{c}\text { TETO } \\
\text { (a) }\end{array}$} & \multirow{2}{*}{$\begin{array}{l}\text { TETO } \\
\text { (b) }\end{array}$} & \multicolumn{2}{|c|}{$\begin{array}{c}\text { Cobertura de } \\
\text { NASF }(\%)\end{array}$} & \multicolumn{2}{|c|}{$\begin{array}{c}\text { Potencial de } \\
\text { Expansão }\end{array}$} \\
\hline & & & & & $\begin{array}{l}\text { TETO } \\
\text { (a) }\end{array}$ & $\begin{array}{l}\text { TETO } \\
\text { (b) }\end{array}$ & (a) & (a) \\
\hline Belém de São & 7 & 0 & 1,4 & 0,77 & 0 & & 1,4 & 0,77 \\
\hline Francisco & & & & & & 0 & & \\
\hline Cedro & 5 & 0 & 1 & 0,55 & 0 & 0 & 1 & 0,55 \\
\hline Mirandiba & 5 & 0 & 1 & 0,55 & 0 & 0 & 1 & 0,55 \\
\hline Salgueiro & 18 & 1 & 3,6 & 2 & 27,77 & 50 & 2,6 & 1 \\
\hline Serrita & 8 & 1 & 1,6 & 0,88 & 62,5 & 113,63 & 0,6 & $-0,12$ \\
\hline Terra Nova & 4 & 0 & 0,8 & 0,44 & 0 & 0 & 0,8 & 0,44 \\
\hline Verdejante & 4 & 0 & 0,8 & 0,44 & 0 & 0 & 0,8 & 0,44 \\
\hline Total & 51 & 2 & 10,2 & 5,66 & 19,60 & 35,33 & 8,2 & 3,66 \\
\hline
\end{tabular}

Fonte: CNES/DATASUS, fevereiro de 2016.

Notas: Teto (a)- considerando a relação de 1 equipe NASF para 5 ESF; Teto (b)considerando a relação de 1 equipe de NASF para 9 ESF.

Na VIII Regional observa-se que o teto de implantação é de 25 NASF considerando o teto mínimo e 14 NASF considerando o teto máximo. A cobertura encontrada foi de $42,97 \%$ 
(teto a) e 77,36\% (teto b). Nos municípios de Cabrobó, Lagoa Grande e Petrolina são necessários a implantação de novos NASF de forma que se atenda aos parâmetros recomendados. Ao todo na regional o potencial de expansão corresponde a 14 equipes considerando o teto mínimo e 3 equipes de acordo com o teto máximo (tabela 3 ).

Tabela 3 - Teto de implantação, cobertura e potencial de expansão do NASF na VIII regional de Saúde da IV Macro do estado de Pernambuco na competência fevereiro de 2016.

\begin{tabular}{|c|c|c|c|c|c|c|c|c|}
\hline \multirow[t]{2}{*}{ Município } & \multirow[t]{2}{*}{$\begin{array}{l}N^{\circ} \text { de } \\
\text { ESF }\end{array}$} & \multirow[t]{2}{*}{$\begin{array}{c}N^{0} \text { de } \\
\text { NASF } 1\end{array}$} & \multirow{2}{*}{$\begin{array}{l}\text { TETO } \\
\text { (a) }\end{array}$} & \multirow{2}{*}{$\begin{array}{l}\text { TETO } \\
\text { (b) }\end{array}$} & \multicolumn{2}{|c|}{$\begin{array}{l}\text { Cobertura de } \\
\text { NASF }(\%) \\
\text { TETO }(\end{array}$} & \multicolumn{2}{|c|}{$\begin{array}{c}\text { Potencial de } \\
\text { Expansão }\end{array}$} \\
\hline & & & & & $\begin{array}{l}\text { TETO( } \\
\text { a) }\end{array}$ & TETO(b) & $\mathbf{a}$ & $\mathbf{b}$ \\
\hline Afrânio & 6 & 0 & 1,2 & 0,67 & 0 & 0 & 1,2 & 0,67 \\
\hline Cabrobó & 11 & 1 & 2,2 & 1,22 & 45 & 81,97 & 1,2 & 0,22 \\
\hline Dormentes & 5 & 0 & 1 & 0,55 & 0 & 0 & 1 & 0,55 \\
\hline Lagoa Grande & 10 & 1 & 2 & 1,11 & 50 & 90,09 & 1 & 0,11 \\
\hline Orocó & 4 & 0 & 0,8 & 0,44 & 0 & 0 & 0,8 & 0,44 \\
\hline Petrolina & 83 & 8 & 16,6 & 9,22 & 48,19 & 86,77 & 8,6 & 1,22 \\
\hline $\begin{array}{l}\text { Santa Maria } \\
\text { da Boa Vista }\end{array}$ & 9 & 1 & 1,8 & 1 & 55,55 & 100 & 0,8 & 0 \\
\hline Total & 128 & 11 & 25,6 & 14,22 & 42,97 & 77,36 & 14,6 & 3,22 \\
\hline
\end{tabular}

Fonte: CNES/DATASUS, fevereiro de 2016.

Por fim, observa-se que na IX regional de saúde o teto de implantação corresponde a 22 equipes considerando o teto a ou 12 NASF de acordo com teto b. A cobertura é de 57,1\% (teto a) e de 102,6\% (teto b). Percebe-se que essa regional possui a maior cobertura de NASF e que o potencial de expansão é de apenas 9 equipes. Contudo, assim como em municípios de outras regionais, é necessário a adequação dos parâmetros de vinculação com a implantação de novas equipes (tabela 4 ).

As coberturas excedentes a $100 \%$ são justificadas pelo fato de que esses municípios estão adequados as normas preconizadas. Exemplificando, no município de Araripina observa-se que o mesmo possui 17 ESF e 2 NASF do tipo 1. Conforme as orientações do MS, essa modalidade pode dar suporte até 9 Equipes, nesse caso, 2 NASF podem oferecer apoio 
até 18 ESF. Os dados encontrados abaixo do valor 1 significa que não precisam ser implantadas novas equipes de NASF.

Tabela 4 - Teto de implantação, cobertura e potencial de expansão do NASF na IX regional de Saúde da IV Macro do estado de Pernambuco na competência fevereiro de 2016.

\begin{tabular}{|c|c|c|c|c|c|c|c|c|}
\hline \multirow[t]{2}{*}{ Município } & \multirow[t]{2}{*}{$\begin{array}{c}\mathbf{N}^{0} \text { de } \\
\text { ESF }\end{array}$} & \multirow[t]{2}{*}{$\begin{array}{c}N^{0} \text { de } \\
\text { NASF } 1\end{array}$} & \multirow{2}{*}{$\begin{array}{c}\text { TETO } \\
\text { (a) }\end{array}$} & \multirow{2}{*}{$\begin{array}{c}\text { TETO } \\
\text { (b) }\end{array}$} & \multicolumn{2}{|c|}{$\begin{array}{l}\text { Cobertura de } \\
\text { NASF }(\%)\end{array}$} & \multicolumn{2}{|c|}{$\begin{array}{l}\text { Potencial de } \\
\text { Expansão }\end{array}$} \\
\hline & & & & & $\begin{array}{l}\text { TETO } \\
\text { (a) }\end{array}$ & $\begin{array}{l}\text { TETO( } \\
\text { b) }\end{array}$ & $\mathbf{A}$ & B \\
\hline Araripina & 17 & 2 & 3,4 & 1,89 & 58,82 & 105,82 & 1,4 & $-0,11$ \\
\hline Bodocó & 11 & 1 & 2,2 & 1,22 & 45,45 & 81,97 & 1,2 & 0,22 \\
\hline Exu & 13 & 2 & 2,6 & 1,44 & 76,92 & 138,88 & 0,6 & $-0,56$ \\
\hline Granito & 3 & 0 & 0,6 & 0,33 & 0 & 0 & 0,6 & 0,33 \\
\hline Ipubi & 11 & 1 & 2,2 & 1,22 & 45,45 & 81,97 & 1,2 & 0,22 \\
\hline Moreilândia & 5 & 0 & 1 & 0,56 & 0 & 0 & 1 & 0,56 \\
\hline Ouricuri & 24 & 3 & 4,8 & 2,67 & 62,5 & 112,36 & 1,8 & $-0,33$ \\
\hline Santa Cruz & 6 & 1 & 1,2 & 0,67 & 83,33 & 149,25 & 0,2 & $-0,33$ \\
\hline Santa & 5 & 1 & 1 & 0,56 & 100 & & 0 & $-0,44$ \\
\hline Filomena & & & & & & 178,57 & & \\
\hline Trindade & 11 & 1 & 2,2 & 1,22 & 45,45 & 81,97 & 1,2 & 0,22 \\
\hline Parnamirim & 8 & 1 & 1,6 & 0,88 & 62,5 & 113,63 & 0,6 & $-0,12$ \\
\hline Total & 114 & 13 & 22,8 & 12,67 & 57,01 & 102,6 & 9,8 & $-\mathbf{0 , 3 3}$ \\
\hline
\end{tabular}

Fonte: CNES/DATASUS, 2016.

\section{Discussões}

A criação do NASF possibilita a ampliação de possibilidades e estratégias de atuação ao incluir profissionais que não estavam inseridos na equipe mínima de saúde da família, representando um grande avanço para a consolidação e fortalecimento do SUS. Gonçalves et al (2015) complementam que esse serviço visa ampliar a abrangência e resolutividade na atenção primária, tratando-se de uma nova proposta de trabalho que se encontra em processo de construção e de efetivação de práticas nos territórios junto a população. 
A inserção desse serviço pelos gestores municipais pode sinalizar um esforço em gerar mudanças no modo de fazer saúde, atuando a partir de um referencial distinto do modelo hegemônico. Entretanto, apenas a sua implantação não é suficiente para garantir a melhoria das condições de saúde da população, uma vez que os fatores determinantes da saúde extrapolam o setor e passam por questões como acesso aos bens e serviços, trabalho, renda, educação, segurança, dentre outros (TOMASI; RIZZOTTO, 2013).

Cabe destacar que o processo de implantação é de iniciativa do gestor municipal que em conjunto com os profissionais que atuam na rede de atenção do município devem identificar as principais necessidades apresentadas pela população bem como a disponibilidade de profissionais que poderão atuar nesse serviço (BRASIL, 2014).

Nesse sentido, destaca-se que os resultados encontrados nessa pesquisa são apenas sugestões de ampliação de NASF nos territórios. A implantação do referido serviço não deve ser realizada considerando apenas o parâmetro quantitativo, uma vez que o investimento na multiplicação de dispositivos na rede de atenção a saúde que tenham esse caráter deve ser realizado a partir de um estudo das necessidades de cada localidade considerando o seu perfil epidemiológico, a disponibilidade e oferta de profissionais e as necessidades da população.

O Ministério da Saúde orienta que a implantação do NASF deve iniciar com a elaboração de um projeto que contenha a análise do território e das necessidades em saúde identificados por vários atores: equipes de atenção básica, população e gestores da saúde (BRASIL, 2014).

Para definição do número de equipes para cada NASF, deve-se procurar uma organização que contemple o menor número de equipes vinculadas, proximidade geográfica entre as equipes e integração entre as necessidades de saúde da população e recursos do território. Essa distribuição permitirá que os profissionais do NASF possam atuar em mais de uma Unidade Básica de Saúde (UBS) ao mesmo tempo, garantindo menor distância e menos tempo para deslocamento entre as unidades, otimizando o desenvolvimento das atividades. Além disso possibilita potencializar as ações a partir de recursos no território, como por exemplo, a realização de atividades coletivas na Academia da Saúde (BRASIL, 2014). 
Sobre a inserção desse serviço na rede de atenção a saúde, Kichel (2014) descreveu que o número de NASF cresceu gradativamente nos primeiros anos de implantação. No primeiro momento, os requisitos eram mais rígidos do que os atuais com o número limitado de equipes de saúde da família para vinculação ao NASF. Assim, pequenos municípios brasileiros não podiam implantar esse serviço, e dessa forma, o Ministério da Saúde revisou os parâmetros no ano de 2012 de modo a contemplar todos com a oferta. O NASF na modalidade 1 pode dar suporte de 5 a 9 ESF, a modalidade 2 de 3 a 4 ESF e a modalidade 3 de 1 a 2 ESF (BRASIL, 2012).

De acordo com o Ministério da Saúde o NASF na modalidade 3 foi criado com o intuito de possibilitar que qualquer município brasileiro possa implantar essa estratégia (BRASIL, 2012).

Em confronto a essas informações, observou-se nessa pesquisa que os parâmetros de vinculação não encontram-se adequados a normatização do MS. Os dados encontrados revelaram que em alguns municípios não há a implantação desse serviço e em outros há um excesso de Equipes de Saúde da Família vinculadas ao NASF. Portanto, verifica-se que há a necessidade de expansão com vistas a um adequado atendimento às equipes existentes.

Nessa perspectiva, Avejonas, et al (2014) relatam que é fundamental que ocorra a adequação as normas previstas, visando ao bom funcionamento e a garantia de ações com qualidade. Ribeiro et al (2014) complementam que a implantação desse serviço com número insuficiente de equipes pode ser um obstáculo à formação de vínculo, gerando conflitos e frustrações aos profissionais pela dificuldade em atender as demandas locais.

Araújo e Galimbertti (2013) corroboram com as informações ao identificarem em sua pesquisa o sofrimento vivido pelos profissionais do NASF no acompanhamento de um número excessivo de equipes. Foi relatado o quanto que a agenda do NASF pode ser desafiadora, pois muitas vezes observou-se que os profissionais não conseguiam se reunir novamente com a ESF.

Também é importante salientar que a ausência de implantação desse serviço dificulta o acesso da população aos profissionais que não estão inseridos na equipe mínima de saúde da 
família e que por esse motivo só teriam contato a partir do encaminhamento para serviços de atenção especializada, gerando assim filas de espera por problemas que poderiam ser resolvidos ou atendidos na própria atenção primária.

Gonçalves et al (2015) corroboram com a ideia de que o NASF promove o acesso da população à assistência em saúde, evitando encaminhamentos para serviços especializados. Os autores descreveram que esse serviço apresenta como objetivo ampliar a abrangência e resolutividade da atenção primária.

Sobre a necessidade de implantação de novas equipes, Santos; Benedetti (2012) destacaram que o NASF apresenta um grande potencial de expansão. Os autores descreveram que o principal fator que pode justificar a baixa implantação seria o receito da gestão na expansão dessas equipes. Os gestores podem ter sido cautelosos, uma vez que ainda não se sabia das potencialidades do NASF e qual a melhor forma de gerenciá-lo.

Patrocínio; Machado e Fausto (2015) também corroboram com a pesquisa destacando que o NASF é uma proposta recente e apresenta potencial de expansão nas localidades em que ainda não se atingiu o quantitativo de NASF de acordo com a regulamentação do Ministério da Saúde.

Na pesquisa de Santos; Benedetti (2012) foram encontradas 1.377 equipes de NASF credenciadas em todo o Brasil no ano de 2011. Nesse ano, observou-se que a maioria dos municípios ainda não tinham implantado esse serviço e entre os que implantaram a maior parcela possuía apenas uma equipe.

No estudo de Patrocínio; Machado e Fausto (2015) foi encontrado que somente em 7 estados o número de equipes encontra-se na faixa recomendada pelo Ministério da Saúde: um estado da região norte (RR), cinco estados da região Nordeste (CE, PB, PE, PI, RN) e um estado da região Centro-Oeste (MS). Os dados mostraram que a expansão do NASF foi expressiva no País, embora desigual no território nacional. Os autores descreveram que em 2011 existiam em todo o país 1.580 NASF,s, sendo 1.415 NASF tipo 1 e 174 NASF tipo 2 com uma razão de 20,3. A razão encontra-se acima da faixa recomendada pelo Ministério da 
Saúde. Em Pernambuco foram encontrados 125 NASF tipo 1 e 11 NASF tipo 2, com uma razão NASF/ESF de 13,7.

Já Silva et al (2014) destacaram que o número de equipes multiprofissionais e cobertura do NASF foram triplicados em 4 anos, sinalizando preocupação em promover mudanças nos níveis de saúde da população. Apesar desse progresso, os autores concordam que é necessário realizar esforços para ampliar o número de ESF e do NASF nos municípios, melhorando não só a cobertura populacional mas também o enfoque em ações que englobem agenda prioritária de problemas de saúde pública.

O processo de implantação do NASF alcança hoje todo o território nacional e tem se adequado as diversas realidades municipais. A proposta de criação aparece da necessidade de apoio às equipes de atenção básica nas suas práticas gerenciais e de atenção à saúde (KICHEL, 2014).

\section{Considerações finais}

A partir dos dados encontrados observou-se que o número de NASF implantados é insuficiente para apoiar as ESF existentes. Dessa forma, verifica-se que há a necessidade de expansão dessas equipes com vistas a adequação das normas preconizadas pelo Ministério da Saúde.

Os critérios de vinculações das Equipes de Saúde da Família (ESF) ao NASF constituem-se como essenciais para o bom desenvolvimento do trabalho, uma vez que o excesso de ESF que necessitam de apoio implicará em uma menor presença dos profissionais apoiadores nessas unidades. Portanto, acredita-se que a adequação das normas possibilita uma melhor atuação desse dispositivo na rede de atenção a saúde favorecendo o atendimento do maior número de necessidades apresentadas no território.

Alguns questionamentos surgem: Que fatores influenciam a maior implantação do NASF em algumas regiões? Será que os gestores têm ciência das necessidades do território e da importância da implantação desse componente na rede de atenção à saúde? Será que de 
fato não há a necessidade de inserção/ampliação desse serviço nos referidos municípios? E para aqueles que possuem mais equipes vinculadas ao NASF além do recomendado, quais as implicações para o processo de trabalho e o cuidado dos usuários?

Nessa perspectiva, destaca-se a necessidade da realização de novos estudos que visem averiguar o grau de implantação do NASF nos estados brasileiros e quais as implicações da inserção ou não desse serviço no processo de trabalho da atenção primária e na atenção as necessidades de saúde da população.

Ademais, destaca-se que as informações obtidas são apenas parâmetros quantitativos que visam conscientizar os gestores da importância da inserção desse componente em acordo com as normatizações. Contudo, vale ressaltar que a implantação desse serviço deve ser realizada após um estudo das necessidades de cada localidade considerando uma análise situacional do território.

\section{Referências}

ANJOS, K. F. et al. Perspectivas e desafios do núcleo de apoio à saúde da família quanto às práticas em saúde. Saúde em Debate, Rio de Janeiro, v. 37, n. 99, p.672-680, out/dez. 2013. Disponível em: < http://www.scielo.br/pdf/sdeb/v37n99/a15v37n99.pdf>. Acesso em: 26 de março de 2016.

ARAÚJO, E. M. D; GALIMBERTTI, P. A. A colaboração interprofissional na Estratégia Saúde da Família. Psicologia \& Sociedade, v.25, n.2, p. 461-468, 2013.

AVEJONAS, M. D. R et al. Inserção e atuação da Fonoaudiologia nos Núcleos de Apoio à Saúde da Família. Codas. São Paulo, vol.26 no.2 mar./abr. 2014. Disponível em: < http://w ww.scielo.br/scielo.php?script=sci_arttext\&lng=pt\&nrm=iso\&tlng=pt\&pid=S2317$17822014000200148>$. Acesso em: 27 de março de 2016.

BRASIL. Ministério da Saúde. Portaria n ${ }^{\circ}$. 154, de 24 de janeiro de 2008 - Cria os Núcleos de Apoio à Saúde da Família. Diário Oficial da União, nº 43, 38-42, 2008.

.Ministério da Saúde. Secretaria de Atenção à Saúde. Departamento de Atenção Básica. Diretrizes do NASF: Núcleo de Apoio à Saúde da Família. Brasília: Ministério da 
Saúde; 2009. 160 p. [Série A. Normas e Manuais Técnicos/ Cadernos de Atenção Básica, n. 27].

.Ministério da Saúde. Portaria n ${ }^{\circ}$ 2.488, de 21 de outubro de 2011. Aprova a Política Nacional de Atenção Básica, estabelecendo a revisão de diretrizes e normas para a organização da Atenção Básica, para a Estratégia Saúde da Família (ESF) e o Programa Agentes Comunitários de Saúde (PACS). Diário Oficial da União, Brasília- DF, seção 1, p. 48-55, 2011.

Ministério da Saúde. Portaria 3.124 de 28 de dezembro de 2012. Redefine os parâmetros de vinculação dos Núcleos de Apoio à Saúde da Família (NASF) Modalidades 1 e 2 às Equipes Saúde da Família e/ou Equipes de Atenção Básica para populações específicas, cria a Modalidade NASF 3, e dá outras providências. Diário Oficial da União, seção 1, p.223, 2012. Disponível em: < http: // bvsms .saude. gov.br/ bvs/ saudelegis/ gm/ 2012/ prt3124_28_12_2012.html>. Acesso em: 10 de abril de 2016.

Ministério da Saúde. Secretaria de Atenção à Saúde. Departamento de Atenção Básica. Núcleo de Apoio à Saúde da Família- Volume 1: Ferramentas para a gestão e para o trabalho cotidiano. Cadernos de Atenção Básica, n. 39, 2014. Disponível em: < http://189.28.128.100/dab/docs/portaldab/publicacoes/caderno_39.pdf >. Acesso em: $10 \mathrm{de}$ abril de 2016.

FITIPALDI, A. L. M; ROMANO, V. F; BARROS, D. C. Nas entrelinhas do olhar: Apoio Matricial e os profissionais da Estratégia Saúde da Família. Saúde em Debate. Rio de Janeiro, v. 39, n. 104, p. 76-87, jan-mar, 2015. Disponível em: < http://www.scielo.br/pdf/sdeb/v39n104/0103-1104-sdeb-39-104-00076.pdf>. Acesso em: 27 de março de 2016.

GONÇALVES, R. M. D. A. et al. Estudo do trabalho em Núcleos de Apoio à Saúde da Família (NASF), São Paulo, Brasil. Rev. bras. Saúde ocupacional, São Paulo, v. 40, n. 131, p. 59-74, 2015. Disponível em: <http://www.scielo.br/pdf/rbso/v40n131/0303-7657-rbso-40131-59.pdf>. Acesso em: 27 de março de 2016.

KICHEL, P. G. Núcleos de apoio à Saúde da Família- NASF: a proposta do apoio matricial na atenção básica. Trabalho de conclusão de curso de Formação em Saúde Coletiva e Educação apresentado a Universidade Federal do Rio Grande do Sul. Brasília- DF, 2014. 59p. Disponível em: < https:// www.lume.ufrgs.br/bitstream/handle/10183/ 114764/000954648.pdf?sequence=1>. Acesso em: 20 de maio de 2016.

LIMA, F. L. C. D. O Núcleo de Apoio à Saúde da Família e alguns dos seus desafios. Saúde e Desenvolvimento, v. 3,n. 2, p. 118-133, jan/jun, 2013. Disponível em: <http://www. grupouninter.com.br/revistasaude/index.php/saudeDesenvolvimento/article/view/144/114>. Acesso em: 28 de março de 2016. 
MAGALHÃES JUNIOR, H. M. Redes de Atenção à Saúde: rumo à integralidade. Revista S aúde em debate, Rio de Janeiro, n.52, p. 15-37, outubro de 2014. Disponível em: <http://cebes.org.br/site/wp-content/uploads/2014/12/Divulgacao-52.pdf >. Acesso em: $28 \mathrm{de}$ março de 2016.

MENDES, E. V. As redes de atenção à saúde. $2^{\text {a }}$. ed. Brasília: Organização Pan-Americana de Saúde, 2011. 549 p. Disponível em: < http://www. conass. org.br/ pdf/ Redes_ de_ Atencao.pdf >. Acesso em: 28 de março de 2016.

E. V. As redes de atenção à saúde. Ciênc. Saúde Coletiva, v. 15, n. 5, p.2297-2305, 2010. Disponível em: < http://www.scielo.br/pdf/csc/v15n5/v15n5a05.pdf>. Acesso em: 28 de março de 2016.

E. V. Comentários sobre as Redes de Atenção à Saúde no SUS. Revista Saúde em debate. Rio de Janeiro, n. 52, p. 38-49, outubro 2014. Disponível em: <http:// cebes. org. br/ site/wp-content/uploads/2014/12/Divulgacao-52.pdf>. Acesso em: 29 de março de 2016.

NASCIMENTO, D. D. G. D.; OLIVEIRA, M. A. D. C. Reflexões sobre as competências profissionais para o processo de trabalho nos Núcleos de Apoio à Saúde da Família. O Mundo da Saúde, São Paulo, v. 34, n.1, p. 92-96, 2010. Disponível em: < http://saocamilosp.br/pdf/mundo_saude/74/12_revisao_reflexoes.pdf>. Acesso em: 29 de março de 2016.

PATROCÍNIO, S. S. S; MACHADO, C. V; FAUSTO, M. C. R. Núcleo de Apoio à Saúde da Família: proposta nacional e implementação em municípios do Rio de Janeiro. Revista Saúde em Debate, Rio de Janeiro, v. 39, n. especial, p. 105-119, dez, 2015. Disponível em: < http://www.scielo.br/pdf/sdeb/v39nspe/0103-1104-sdeb-39-spe-00105.pdf>. Acesso em: 20 de maio de 2016.

PERNAMBUCO. Secretaria Estadual de Saúde. Plano Diretor de Regionalização. Pernambuco, 2011. Disponível em: <http:// portal.saude.pe.gov.br/sites/portal.saude.pe. gov.br/files/pdrconass-versao_final1.doc_ao_conass_em_jan_2012.pdf $>$. Acesso: em $10 \mathrm{de}$ abril de 2016.

RIBEIRO, M. D. A. et al. Avaliação da atuação do Núcleo de Apoio à Saúde da Família. Revista Brasileira em Promoção da Saúde. Fortaleza, 27(2): 224-231, abr./jun., 2014. Disponível em: < http://ojs.unifor.br/index.php/RBPS/article/view/2426>. Acesso em: 30 de março de 2016.

SANTOS, S. F. D. S. D.; BENEDETTI, T. R. B. Cenário de implantação do Núcleo de Apoio a Saúde da Família e a inserção do profissional de Educação Física. Rev Bras Ativ Fis e Saúde, Pelotas/RS, v. 17, N.3, p. 188-194, 2012. Disponível em: <http://periodicos.ufpel. edu.br/ojs2/index.php/RBAFS/article/view/1857/1697>. Acesso em: 30 de março de 2016. 
SILVA, M. A. P et al. Atenção Básica em Alagoas: expansão da Estratégia Saúde da Família, do Nasf e do componente alimentação/nutrição. Revista Saúde em Debate, Rio de Janeiro, v. 38, n. 103, p. 720-732, out-dez, 2014. Disponível em: <http://www.scielo.br/pdf/sdeb/ v38n103/0103-1104-sdeb-38-103-0720.pdf>. Acesso em: 21 de maio de 2016.

TOMASI, A. R. P.; RIZZOTTO, M. L. F. Análise da distribuição e composição profissional dos Núcleos de Apoio à Saúde da Família no Paraná. Saúde em Debate. Rio de Janeiro, v. 37, n. 98, p. 427-436, jul/set, 2013. Disponível em: < http://www.scielo.br/pdf/sdeb/v37n98/a06v37n98.pdf>. Acesso em: 30 de março de 2016.

\section{Como citar este artigo (Formato ABNT):}

PARENTE, Alaine S.; MESQUITA, Fabíola O. S.; SARMENTO, Sued S. Análise da distribuição e cobertura do NASF na IV Macrorregião de Saúde do Estado de Pernambuco.. Id on Line Revista Multidisciplinar e de Psicologia, Julho de 2017, vol.11, n.36, p.435-453. ISSN: 1981-1179.

Recebido: 26.07.2017

Aceito: 29.07 .2017 